\title{
Wave Phenomena in High-Voltage Windings of Transformers
}

\author{
S. BANASZAK AND K.M. GAWRYLCZYK* \\ West Pomeranian University of Technology, Department of Electrotechnology and Diagnostics \\ W. Sikorskiego 37, 70-313 Szczecin, Poland
}

\begin{abstract}
Determination of mechanical condition of transformer active part can be performed utilizing frequency response of the windings. Many computing models were developed to evaluate behavior of the winding in wide frequency range using different numerical methods. Most of them utilizes finite elements, assuming axial symmetry, if possible. In other cases we need to use a three-dimensional model, which results in a significant reduction in the accuracy of modeling the geometry of the system, as well as the penetration of electromagnetic field into conductors. From a practical point of view, using computer of average performance, the windings can be modeled with at most 10 turns. From this reason mixed models are proposed, where the electric parameters of the winding are concentrated in the substitute $R L C$ elements. Such models are known as "lumped parameter models". Since the parameters of real winding are distributed, the question arises concerning the necessity of taking into account wave phenomena in them. The method allowing this is known as "transmission line method". Measurements of frequency response are used in industry. There are still many problems with interpretation of test results. Computer modeling may be a helpful tool allowing to understand relation between the geometry of a winding and its frequency response. To fulfill this, models should give similar response as a real measurement. The paper describes a modified transmission line method used for modeling of a transformer winding's frequency response. There is described the model, its parameters, and exemplary solution compared to test data.
\end{abstract}

DOI: $10.12693 /$ APhysPolA.125.1335

PACS: 84.37.+q, 03.50.De, 41.20.-q, 84.40.Az

\section{Introduction}

Frequency response (FR) measurements of transformer windings are used in industrial practice as a method for determination of their mechanical condition [1]. Such information is important for asset management of distribution companies, because only the complete information on technical condition of transformers population, together with information on each unit's importance in the system, leads to economically and technically reasonable management. Different high current events, over-voltages, and insulation deterioration may lead to loosening of original winding clamping and subsequently to deformations of windings. Also a core may lose its mechanical integrity over time. FR measurements have been introduced into practice as frequency response analysis (FRA) method.

The transformer winding can be described by a set of local capacitances, self and mutual inductances and resistances. Every change in winding geometry leads to change of these parameters, which influences the shape of the transfer function. The analysis of frequency response measurements results is based on comparison of data presented usually as sine signal damping along frequency spectrum in logarithmic scale. Such curve can be compared to results recorded for a transformer in time intervals, between phases, between twin or sister units or with help of computer models. However, there are still many problems with interpretation of test results

*corresponding author; e-mail: kmg@zut.edu.pl in cases where compared curves are not similar. Due to insufficient number of verified measurements on units with known deformations it is hard to determine if and what problem there is in the winding. A helpful tool may be computer modeling of FR. After obtaining the model giving similar result as a real object it is possible to introduce forced deformations and find out its effect on the FR curve.

The paper describes a new approach to FR modeling presented on the example of few discs belonging to HV-winding, dismounted from the real transformer and examined without the core.

\section{Model construction and computation of lumped parameters}

An approach basing on networks built on lumped parameters of the winding obtained using approximate formulae is known since many years [2]. However, the results were not satisfactory. In our approach the spiral winding is divided on single wires, each of them lying on a $2 \mathrm{D}$ plane (Fig. 1).

The lumped parameters may be calculated on the base of analytic formulae, as in [3], which still do not include skin effect and proximity effect. From this reason in our work the lumped parameters are calculated using finite elements modeling (FEM), wherein each parameter concerns single wire. The contributions of all other wires are also taken into account. Using this parameters the complete network model can be created. The RLC-network model was shown in our earlier works [4]. The results obtained with such models were good upon driving frequency of $1 \mathrm{MHz}$. With such high frequency, transmission line effects become apparent, as the length 


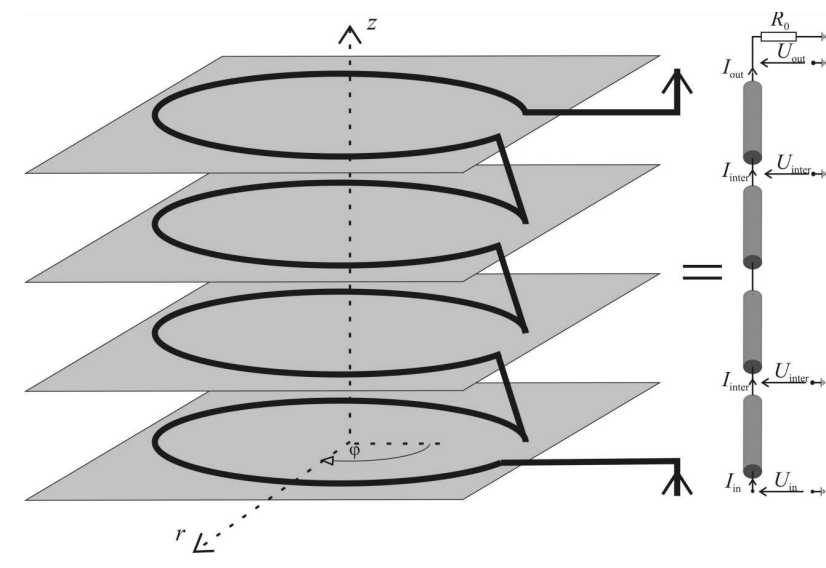

Fig. 1. Schematic illustration of model construction.

of the winding becomes comparable to the electromagnetic wave length. From this reason transmission line method (TLM) was adapted for calculations of lumped parameter network model.

A numerical model of the winding can be realized with commercial FEM-package. Preparation of computer models simulating real phenomena needs choosing approach which leads to good effects with limited software and hardware possibilities.

Usually many simplifications and limitations are introduced into model parameters, to make it possible to perform calculations. In modeling of transformers' windings it is necessary to choose whether $2 \mathrm{D}$ or $3 \mathrm{D}$ model shall be introduced. The first one is related with many limitations, but allows to perform detailed calculations. The second one allows consideration of the real geometry, however current technical possibilities make its results very inaccurate or even impossible to obtain. For exact computation of winding's geometry the three-dimensional model should be used.

In the work [5] very simplified model was used, without analysis of separate wires. In our earlier work [6] there may be found that with nowadays computers the 3D-models containing only 10 wires may be solved, because due to high driving frequency of over $1 \mathrm{MHz}$ the solution has to be carried out using very small finite elements. In practical application there is the need to model windings consisting of several hundred wires. From this reason an approach basing on models with two-dimensional symmetry is proposed. It is obvious that such models enable calculation of only such deformations inside winding, that the cylindrical symmetry is retained.

The next step in modeling is to determine necessary parameters which have to be simulated, to ensure good conformity of model and its physical representation. In discussed case there are considered parameters which affect frequency response and are related to geometry of the winding. There were used self and mutual inductances, capacitances, and resistances, with taking into account skin effects and proximity effects, as well as material properties of all elements.
Another problem is reference to environment. The real object used for creation of described model is placed in laboratory with some influence of surrounding elements. Especially its capacitances may vary from theoretical calculations. As boundary condition of FEM model the ballooning method is used, which reveals better behavior than Dirichlet's or Neumann's boundary conditions. However, the cutoff of the region is obviously a simplification leading to calculation errors.

For FEM calculations commercial package - ANSYS Maxwell was chosen. This program calculates impedance matrices used in network models. Self- and mutual capacitances determination is based on solving given electrostatic field equation

$$
\nabla \cdot\left(\varepsilon_{\mathrm{r}} \varepsilon_{0} \nabla \Phi(r, z)\right)=-\rho
$$

where $\Phi$ is a scalar potential of electric field inducted with charge having its density $\rho, \varepsilon_{0}$ and $\varepsilon_{\mathrm{r}}$ are vacuum permittivity and relative environment permittivity.

To obtain self- and mutual inductances there was solved 2D electromagnetic field equation taking into account eddy currents effect

$$
\nabla \times \frac{1}{\mu}(\nabla \times \boldsymbol{A})+\mathrm{j} \omega \gamma \boldsymbol{A}=\boldsymbol{J}_{\mathrm{s}},
$$

where $\boldsymbol{A}$ is a magnetic vector potential, $\omega$ - supply current pulsation, $\mu$ and $\gamma$ are magnetic permeability of environment and its conductivity. $\boldsymbol{J}_{\mathrm{s}}$ is current density in windings. The induction values are obtained from magnetic field energy $W_{\mathrm{AV}}$ calculated from two models $i$ and $j$ with supply current having peak value $I_{\text {Peak }}$ in given turns

$$
\begin{aligned}
& W_{\mathrm{AV}}=\frac{1}{4} \int \boldsymbol{B}_{i} \cdot \boldsymbol{H}_{j}^{*} \mathrm{~d} V, \\
& L_{i j}=\frac{4 W_{\mathrm{AV}}}{I_{\text {Peak }}^{2}}=\int \boldsymbol{B}_{i} \cdot \boldsymbol{H}_{j} \mathrm{~d} \Omega .
\end{aligned}
$$

$\boldsymbol{B}$ and $\boldsymbol{H}$ are vectors of induction and field intensity of magnetic field, having two spatial components in analyzed case. Self- and mutual capacitances have been obtained by energizing proper turns with voltage and calculating electric field energy $W_{i j}$ :

$$
\begin{aligned}
& W_{i j}=\frac{1}{2} \int_{\Omega} \boldsymbol{D}_{i} \cdot \boldsymbol{E}_{j} \mathrm{~d} \Omega, \\
& C_{i j}=\frac{2 W_{i j}}{V^{2}}=\int_{\Omega} \boldsymbol{D}_{i} \cdot \boldsymbol{E}_{j} \mathrm{~d} \Omega .
\end{aligned}
$$

$\boldsymbol{D}$ and $\boldsymbol{E}$ are vectors of electric induction and electric field intensity, having two components in analyzed case. Turns of resistance values were received from active power losses $P$ in them due to current flow having density $\boldsymbol{J}$ :

$$
\begin{aligned}
& P=\frac{1}{2 \gamma} \int \boldsymbol{J} \cdot \boldsymbol{J}^{*} \mathrm{~d} \Omega, \\
& R=\frac{2 P}{I_{\text {Peak }}^{2}}=\frac{\int \boldsymbol{J} \cdot \boldsymbol{J}^{*} \mathrm{~d} \Omega}{\gamma I_{\text {Peak }}^{2}}=\frac{\int \boldsymbol{J} \cdot \boldsymbol{J}^{*} \mathrm{~d} \Omega}{\gamma},
\end{aligned}
$$

where $I_{\text {Peak }}$ is supply current peak value and $\gamma$ - turn conductivity. Discussed model is presented in Fig. 2. 


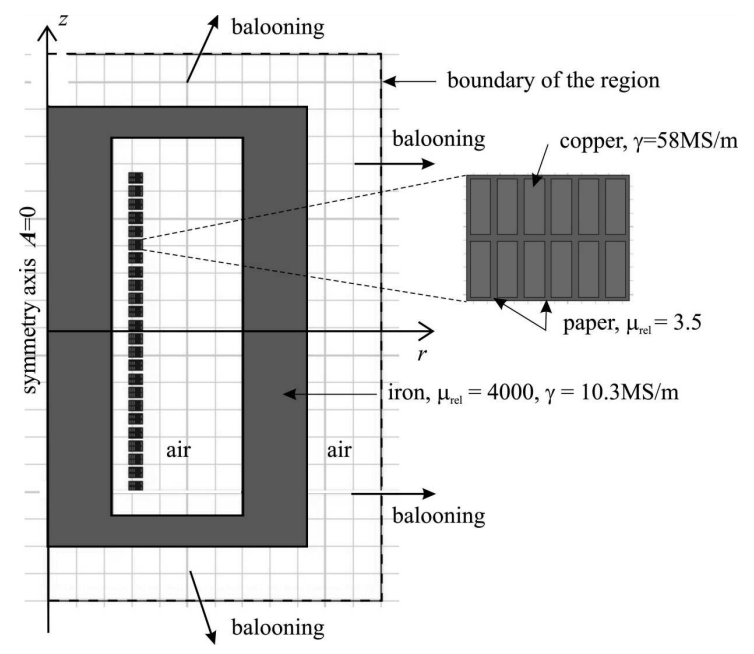

Fig. 2. Model created in FEM software with real dimensions and material properties.

A very similar treatment of this problem may be found in [7]. The analyzed area was discretized with about $64 \mathrm{k}$ triangular elements. Calculations were carried out for six frequencies from $100 \mathrm{~Hz}$ until $10 \mathrm{MHz}$. Skin effect at high frequency is clearly visible not only in the values of the winding resistance, but also in the mutual and own inductances.

\section{Transmission line method}

The frequency of excitation current when using FRA reaches $10 \mathrm{MHz}$ and the length of $\mathrm{HV}$ transformer winding is several hundred meters, so it is comparable to wave length $300 \mathrm{~m}$ at the frequency of $1 \mathrm{MHz}$. From this reason better results may be obtained by considering the wave effects. Distributed parameter networks were studied many years ago. Transmission line models are included in the most popular simulators, as SPICE. The history of transmission line models and a model of $\mathrm{AC}$ motor may be found in [8].

Presented research is based on the conception presented by [9]. Each turn from Fig. 1 lies in separate plane and can be replaced by the element of transmission line. Because the turns are placed at one 2D-plane $(r-\phi)$, the use of two-dimensional models in finite element analysis is possible. The interconnection of turns forming complete winding will be done later, in a network model. Behavior of transmission line elements describe well known telegraph equations

$$
\begin{aligned}
& \frac{\mathrm{d} \boldsymbol{U}}{\mathrm{d} x}=-\boldsymbol{Z} \cdot \boldsymbol{I}, \quad \boldsymbol{Z}=\boldsymbol{R}+\mathrm{j} \omega \boldsymbol{L}\left[\frac{\Omega}{\mathrm{m}}\right], \\
& \frac{\mathrm{d} \boldsymbol{I}}{\mathrm{d} x}=-\boldsymbol{Y} \cdot \boldsymbol{U}, \quad \boldsymbol{Y}=\mathrm{j} \omega \boldsymbol{C}\left[\frac{\mathrm{S}}{\mathrm{m}}\right] .
\end{aligned}
$$

Since the transmission line elements are coupled to each other, the terms of impedance and admittance matrices $\boldsymbol{Z}$ and $\boldsymbol{Y}$ contain mutual inductances and capacitances. This causes obstacles when solving equation system (6). It may be helpful to use a modal transformation described in [10]. Therefore matrices $\boldsymbol{Z}$ and $\boldsymbol{Y}$ are decomposed to their diagonal form

$$
\boldsymbol{Z}=\boldsymbol{Q}_{\mathrm{u}} \cdot \boldsymbol{Z}_{\mathrm{d}} \cdot \boldsymbol{Q}_{\mathrm{i}}^{-1}, \quad \boldsymbol{Y}=\boldsymbol{Q}_{\mathrm{i}} \cdot \boldsymbol{Y}_{\mathrm{d}} \cdot \boldsymbol{Q}_{\mathrm{u}}^{-1},
$$

where for determination of both matrices, $\boldsymbol{Q}_{\mathrm{i}}$ and $\boldsymbol{Q}_{\mathrm{u}}$ two separate eigendecompositions are proposed

$$
\begin{aligned}
& \boldsymbol{Z} \cdot \boldsymbol{Y}=\boldsymbol{Q}_{\mathrm{u}} \cdot \boldsymbol{Z}_{\mathrm{d}} \cdot \boldsymbol{Y}_{\mathrm{d}} \cdot \boldsymbol{Q}_{\mathrm{u}}^{\mathrm{T}}, \\
& \boldsymbol{Y} \cdot \boldsymbol{Z}=\boldsymbol{Q}_{\mathrm{i}} \cdot \boldsymbol{Y}_{\mathrm{d}} \cdot \boldsymbol{Z}_{\mathrm{d}} \cdot \boldsymbol{Q}_{\mathrm{i}}^{\mathrm{T}} .
\end{aligned}
$$

Presented approach gives better numerical stability of algorithm, versus this presented in [9].

Decomposition (8) allows to decouple telegraph equations in the following way:

$$
\begin{gathered}
\frac{\mathrm{d} \boldsymbol{U}}{\mathrm{d} x}=-\boldsymbol{Q}_{\mathrm{u}} \cdot \boldsymbol{Z}_{\mathrm{d}} \cdot \boldsymbol{Q}_{\mathrm{i}}^{-1} \cdot \boldsymbol{I} \rightarrow \boldsymbol{Q}_{\mathrm{u}}^{-1} \cdot \frac{\mathrm{d} \boldsymbol{U}}{\mathrm{d} x} \\
=-\boldsymbol{Z}_{\mathrm{d}} \cdot \boldsymbol{Q}_{\mathrm{i}}^{-1} \cdot \boldsymbol{i} \rightarrow \frac{\mathrm{d} \boldsymbol{U}_{\mathrm{m}}}{\mathrm{d} x}=-\boldsymbol{Z}_{\mathrm{d}} \cdot \boldsymbol{I}_{\mathrm{m}}, \\
\frac{\mathrm{d} \boldsymbol{I}}{\mathrm{d} x}=-\boldsymbol{Q}_{\mathrm{i}} \cdot \boldsymbol{Y}_{\mathrm{d}} \cdot \boldsymbol{Q}_{\mathrm{u}}^{-1} \cdot \boldsymbol{U} \rightarrow \boldsymbol{Q}_{\mathrm{i}}^{-1} \cdot \frac{\mathrm{d} \boldsymbol{I}}{\mathrm{d} x} \\
=-\boldsymbol{Y}_{\mathrm{d}} \cdot \boldsymbol{Q}_{\mathrm{u}}^{-1} \cdot \boldsymbol{u} \rightarrow \frac{\mathrm{d} \boldsymbol{I}_{\mathrm{m}}}{\mathrm{d} x}=-\boldsymbol{Y}_{\mathrm{d}} \cdot \boldsymbol{U}_{\mathrm{m}},
\end{gathered}
$$

where $\boldsymbol{U}_{\mathrm{m}}, \boldsymbol{I}_{\mathrm{m}}$ mean modal voltages and currents. The solution for modal values is carried out in known way, producing a chain matrix of single element $k$ of a transmission line

$$
\boldsymbol{A}_{m, k}=\left[\begin{array}{cc}
\operatorname{ch} \beta_{k} l & Z_{\mathrm{c}, k} \operatorname{sh} \beta_{k} l \\
\frac{1}{Z_{\mathrm{c}, k}} \operatorname{sh} \beta_{k} l & \operatorname{ch} \beta_{k} l
\end{array}\right],
$$

where $\beta_{k}=\sqrt{Z_{\mathrm{d} k, k} Y_{\mathrm{d} k, k}}$ and $Z_{\mathrm{c}, k}=\sqrt{\frac{Z_{\mathrm{d} k, k}}{Y_{\mathrm{d} k, k}}}$.

After solution of telegraph equations the modal voltages and currents can be transformed to their original values, and the equations system for solving of original currents and voltages is established.

\section{Model verification}

Presented model has been verified with mentioned winding coming from the real transformer (Fig. 3).

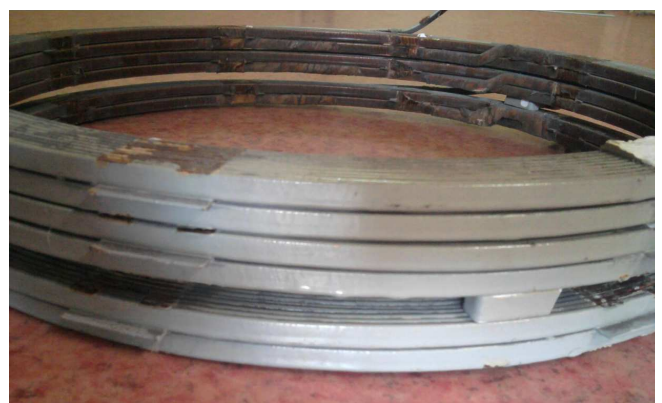

Fig. 3. Six coils of the real winding used for modeling, with visible axial shift.

A measurement of FR has been taken with commercial device Omicron FRAnalyzer. Test results have been compared to simulations. The comparison is presented in Fig. 4.

The shape of FR curve of simulations taken with described TLM model is similar to measurement. It gives 


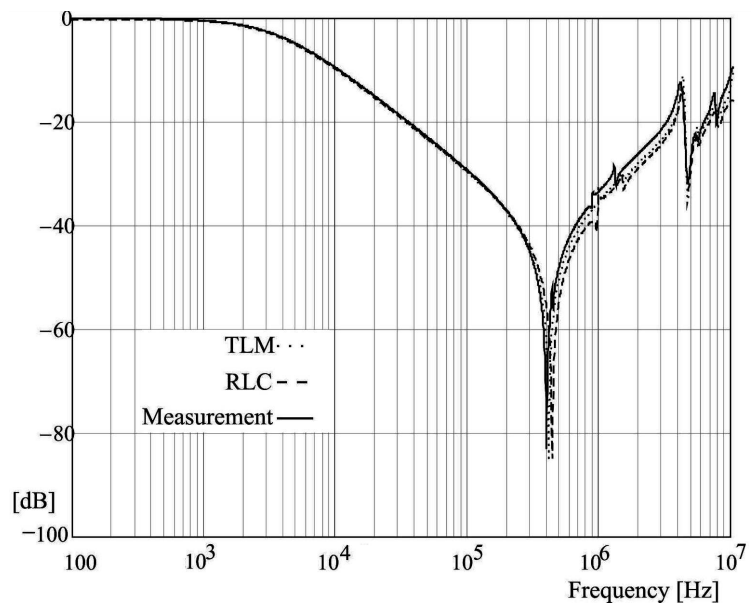

Fig. 4. Results of measurement and simulation with two methods.

more exact values of resonance frequencies than standard RLC model. It shows good conformity up to $10 \mathrm{MHz}$. Visible differences, if compared to test results, are coming from non-perfect conditions of measurement and real winding's geometry, which was not ideally symmetrical.

\section{Summary}

The paper presents the possibility of modeling frequency response of a winding with using the new approach of transmission line method. Described model shows good conformity to real measurements. Also the simulations done for other windings are showing good agreement of results, similarly to example from Fig. 3. It seems that such way of windings modeling might be used for detailed analysis of relation between the geometry of winding and its frequency response. It might be especially used to find the influence of deformations and other faults in the winding on the changes in FR curve shape - frequency range, damping and the character of FR curve modification.

\section{Acknowledgments}

The research was financed by Polish National Science Center, grant no. N N510 698240 titled "The algorithm for identification of transformer winding deformations on the base of frequency response measurements".

\section{References}

[1] International standard IEC 60076-18, Power transformers - Part 18: Measurement of freq7uency response, 2012.

[2] E. Bjerkan, Ph.D. Thesis, Norwegian University of Science and Technology, 2005.

[3] M. Eslamian, B. Vahidi, IEEE Trans. Power Deliv. 27, 2326 (2012).
[4] Sz. Banaszak, K.M. Gawrylczyk, in: Electrical Engineering, Vol. 73, Ed.: R. Nawrowski, Poznań University of Technology, Poznań 2013, p. 107.

[5] Z. Azzouz, A. Foggia, L. Pierrat, G. Meunier, IEEE Trans. Magn. 29, 1407 (1993).

[6] Sz. Banaszak, K.M. Gawrylczyk, in: Computer Application in Electrical Engineering, Poznań University of Technology, Poznań 2010, p. 132.

[7] H. Wang, K.L. Butler, IEEE Trans. Power Deliv. 16, 422 (2001).

[8] G. Skibinski, R. Kerkman, D. Leggate, J. Pankau, D. Schlegel, in: Proc. IEEE Applied Power Conf., Vol. 2, Piscataway 1998, p. 1021.

[9] H. de Gersem, O. Henze, T. Weiland, A. Binder, Compel 29, 23 (2010).

[10] D.J. Wilcox, W.G. Hurley, T.P. McHale, M. Conlon, IEEE Proc.-C 139, No. 6 (1992). 